\title{
The outcome of newborns admitted to kangaroo mother care units at regional hospitals in KwaZulu-Natal, South Africa
}

\author{
W Benguma, ${ }^{1}$ FCPaed; N Khan, ${ }^{1}$ FCPaed, Cert Neonatal; N H McKerrow, ${ }^{1,2}$ MMed (Paed), PG Dip Int Res Ethics \\ ${ }^{1}$ Department of Paediatrics and Child Health, Nelson R Mandela School of Medicine, University of KwaZulu-Natal, Durban, South Africa \\ ${ }^{2}$ KwaZulu-Natal Department of Health, Pietermaritzburg, South Africa
}

Corresponding author: W Benguma (wafabenguma@gmail.com)

\begin{abstract}
Background. Kangaroo mother care (KMC) is a common modality of care for low birthweight and preterm newborns, with good long-term outcomes at low cost. However, little is known about the short-term outcomes of babies during their stay in a KMC unit. Objective. To describe the profile and outcome of newborn babies admitted to KMC units.

Method. A retrospective chart review was undertaken of babies admitted to the KMC units of two Durban hospitals over a two-year period. All babies with birthweights below $2000 \mathrm{~g}$ admitted to the KMC units for the first time during this period were included. Poor outcome was defined as a death in the KMC unit or need for readmission to the neonatal nursery.

Result. Two hundred and twenty-four newborns were included in the study. The median maternal age was 25 years. The newborns had a median gestational age of 32 weeks, median birthweight of $1500 \mathrm{~g}$, median KMC unit admission weight of $1600 \mathrm{~g}$, and median age on admission to the KMC units of 9.5 days. Twenty-six percent of babies had a poor outcome, including seven deaths. Significant factors associated with a poor outcome included a birth or admission weight to KMC units below 1500 g, HIV-negative mothers; and abnormal temperature or blood glucose levels.

Conclusion. Seventy-four percent of babies admitted to KMC were discharged home after an uneventful stay. Poor outcomes were associated with a birth or KMC admission weight below $1500 \mathrm{~g}$ and an abnormal temperature or blood glucose level while admitted to the KMC unit.
\end{abstract}

S Afr J Child Health 2021;15(2):83-88. https://doi.org/10.7196/SAJCH.2021.v15.i1.1739

Although there has been a significant decline in the under-five mortality rate (U5MR) in South Africa (SA) over the past decade, ${ }^{[1]}$ the number of neonatal deaths and the neonatal mortality rate (NMR) remain unchanged. ${ }^{[2]}$ Globally, neonatal deaths account for $47 \%$ of under-five deaths, with $35 \%$ of these being attributed to premature birth. ${ }^{[3]}$ The situation in SA is no different, and almost $30 \%$ of under-five deaths occur in the neonatal period. ${ }^{[1]}$

Preterm and low birthweight (LBW) babies are a major contribution to neonatal mortality and morbidity requiring access to expensive and complex modalities of care. In many low- and middle-income countries, these are not available, and effective low-cost alternatives such as kangaroo mother care (KMC) are required. ${ }^{[4]}$

KMC was established in 1978 in Colombia ${ }^{[5]}$ to cater for preterm newborns at a relatively low cost in a context characterised by overcrowding, nosocomial infection and lack of incubators. Since then, the model has been adopted by many low-, middle- and highincome countries, including SA. ${ }^{[6]}$

$\mathrm{KMC}$ is proven as an inexpensive and effective model of newborn care associated with lower rates of hospital-associated infection, better weight gain and shorter hospital stays. ${ }^{[7]}$ While much is known about the longer-term outcomes ${ }^{[8]}$ and impact of KMC on neonatal mortality, little is known about the short-term need for babies in KMC to be readmitted to a neonatal intensive care unit (NICU). The present study was undertaken to describe the morbidity and mortality of babies admitted to a KMC unit.

\section{Method}

A retrospective review was undertaken of the records of babies admitted to the KMC units of two regional hospitals in Durban, KwaZulu-Natal (KZN) (Mahatma Gandhi Memorial (MGMH) and R K Khan (RKKH) hospitals) over the two-year period from 1 January 2015 to 31 December 2016. All babies with a birthweight below 2 $000 \mathrm{~g}$, who were admitted to the KMC units for the first time during the study period, were eligible for inclusion in the study. Babies with congenital malformations were excluded and only the first admission was reviewed for babies who were admitted to the KMC unit more than once. The primary outcome for the study was discharge, death or readmission of the newborn to the neonatal nursery.

The KMC unit in MGMH has six beds with one nurse per six beds while that in RRKH has eight beds with one nurse per eight beds. Newborns are admitted for weight gain once they are off respiratory support and tolerating all feeds via gastric tube, and are discharged once they have reached a weight of $1700 \mathrm{~g}$ provided they are stable, tolerating full feeds, maintaining their temperature and growing well.

Eligible newborns were identified from the KMC unit admission and discharge register and their clinical records were retrieved from the medical registry in each hospital. Data were extracted from each clinical record and captured onto an Excel (Microsoft Corp., USA) spreadsheet for further analysis. This included maternal demographic, pregnancy and delivery data as well as neonatal birth history, demographic characteristic and clinical data from two periods - admission to the nursery and stay in the KMC units. SPSS version 25 (IBM Corp., USA) was used for statistical analysis, which involved descriptive statistics for demographic data and a $\chi^{2}$ test for categorical variables. A significance level of $p<0.05$ was used. 
The analysis included a description of mothers and newborns as well as a bivariate analysis comparing babies with an uneventful stay in the KMC units with those who had a poor outcome, i.e. they died or required readmission to the neonatal nursery.

Criteria for a poor outcome and readmission to the neonatal nursery included:

- feeding intolerance: any feeding difficulty including poor sucking, refusal or regurgitation of feeds

- neonatal jaundice: total serum bilirubin above the phototherapy line on the 2006 South African Neonatal Academic Hospital Guideline Chart

- anaemia: $\mathrm{Hb}<10 \mathrm{~g} / \mathrm{dL}$ or a symptomatic baby with a higher $\mathrm{Hb}$ level

- sepsis based on:

- clinical features - core temperature $>38.5^{\circ} \mathrm{C}$ or $<36.0^{\circ} \mathrm{C}$; tachycardia or bradycardia; tachypnoea

- laboratory findings - C-reactive protein (CRP) >1 mg/L; elevated or depressed total leukocyte count

- positive culture

- hypoglycaemia: blood glucose $<2.6 \mathrm{mmol} / \mathrm{L}$

- hyperglycaemia: blood glucose $>7.5 \mathrm{mmol} / \mathrm{L}$.

As this was a retrospective chart review, no consent was obtained from parents of the neonates. Ethical approval was granted by the Biomedical Research Ethics Committee of the University of KwaZulu-Natal (BREC ref. no. BE455/17).

\section{Results}

During the study period, 615 newborns were admitted to the KMC units in both hospitals - 358 to MGMH and 257 to RKKH (Fig. 1). Clinical records were not found for 306 (49.7\%) newborns (196 at MGMH and 110 at RKKH) but were found for 309 (50.3\%) newborns (162 at MGMH and 147 at RKKH). Eighty-five newborns did not meet the inclusion criteria (72 with a birthweight above $2000 \mathrm{~g}$ and 13 with a congenital malformation) and 224 newborns were included in the study. Of the newborns included in the study, 166 (74.1\%) had an uneventful stay in the KMC unit; $51(22.8 \%)$ newborns required readmission to the neonatal nursery; and seven $(3.1 \%)$ died while in the KMC unit. In view of the small number of babies with an adverse

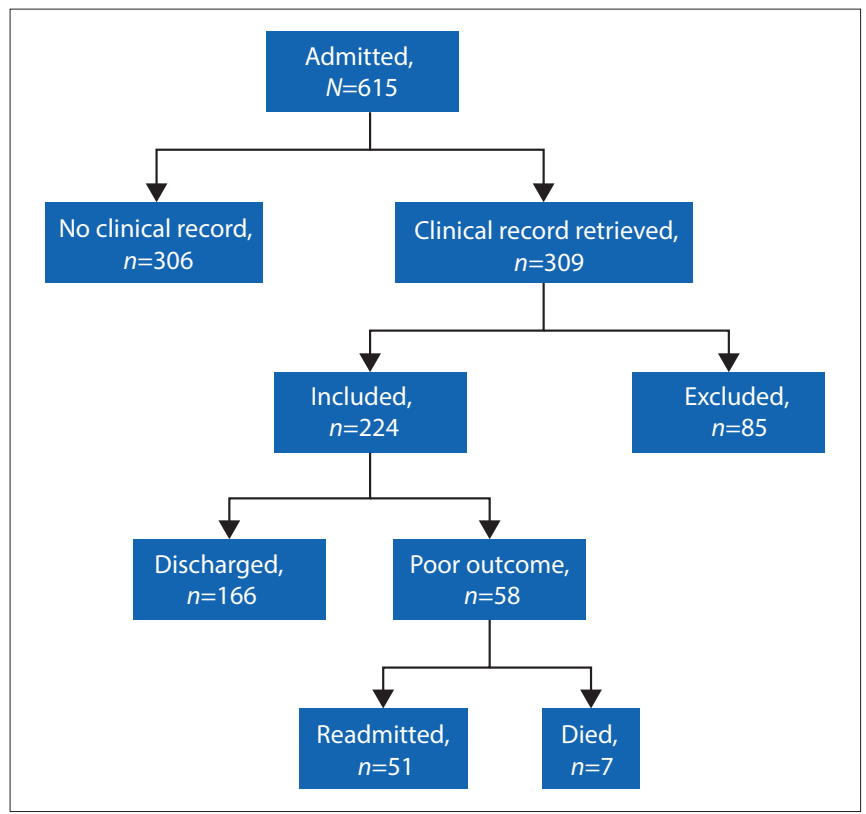

Fig. 1. Sampling strategy and outcomes. (KMC= kangaroo mother care.) outcome when disaggregated to the individual hospitals, the data from the two hospitals were combined for further analysis.

Table 1 depicts the characteristics of the newborns included in the study. An equal number of newborns were delivered vaginally or by caesarean section. Although birthweights ranged from 800 - $1900 \mathrm{~g}$, most newborns had a birthweight between 1000 and $2000 \mathrm{~g}$; a gestational age above 30 weeks; an Apgar score above 7/10; and did not require resuscitation. During their stay in the neonatal nursery, most newborns had neonatal sepsis; received at least first-line antibiotics; and required some respiratory support. Very few had an abnormal temperature or blood glucose level.

Characteristics with a statistically significant association with a poor newborn outcome included birthweight $<1000 \mathrm{~g}(53.0 \%$; $p=0.003)$ and an abnormal temperature $(54.0 \% ; p=0.005)$ and an abnormal blood glucose level $(43.2 \%$; $p=0.035)$.

Table 2 shows the characteristics of mothers in the study. Twentyfour (10.7\%) were less than 19 years of age, and 14 (6.3\%) were above the age of 35 years. A poor outcome was more common among newborns of young mothers (33.4\%) and lowest among those of older mothers; however, this difference was not statistically significant $(p=0.645)$. More than a third $(36.6 \%)$ of mothers were primigravidas and only three were grand multiparous. Most mothers received antenatal care $(86.6 \%)$ and, of these, $24.8 \%$ had newborns with a poor outcome, compared with $33.4 \%$ of those who had not received antenatal care. Maternal HIV status was found to be statistically significant $(p=0.017)$, although unexposed newborns had a higher rate of poor outcomes compared with exposed newborns (31.2\% v. 16.5\%). While pregnancy-related problems were common, chronic maternal diseases were associated with a higher rate of poor outcome; however, the numbers were small and not statistically significant.

Neither the age nor the presence of problems on admission to KMC were associated with poor newborn outcome. However, an abnormal temperature or blood glucose level or a new problem arising during admission to the KMC all had a statistically significant association with poor outcome (Table 3). All newborns were admitted to KMC for weight gain but for $38(17 \%)$ this was compromised by the development of complications including feed intolerance, anaemia and other clinical problems. These newborns had a significantly higher rate of poor outcomes The frequency of a poor outcome increased with decreasing weight on admission to KMC, and a poor outcome was more common among newborns who had an abnormal temperature or blood glucose level during their admission to the KMC unit.

Table 4, with the final diagnoses of the treating clinicians, shows that the main reason for readmission to the neonatal nursery was neonatal sepsis $(60.6 \%)$, and the main cause of death was aspiration pneumonia (42.8\%).

\section{Discussion}

A quarter of babies admitted to the KMC units had a poor outcome - $51(22.7 \%)$ required readmission to the nursery and 7 (3.1\%) died, which is similar to other reports from southern Africa, e.g. Blencowe ${ }^{[21]}$ reported a readmission rate of $15.8 \%$ in Malawi in 2005 and Kambarami et al. ${ }^{[18]}$ reported a $28 \%$ readmission rate in Zimbabwe in 1999, although neither reported on the number of deaths.

The reasons for readmission to the nursery from the KMC unit differed between countries, with sepsis accounting for 55\% of readmissions in KZN while $45 \%$ of readmissions in Zimbabwe were attributed to respiratory problems. Maternal problems (2.0\% v. $5.0 \%)$, 


\begin{tabular}{|c|c|c|c|c|}
\hline Indicator & Total $(N=224), n(\%)$ & Discharge $(N=166), n(\%)$ & Poor outcome $(N=58), n(\%)$ & $p$-value \\
\hline Mode of delivery & & & & 0.643 \\
\hline NVD & $110(49.0)$ & $80(72.7)$ & $30(27.3)$ & \\
\hline CS & $114(51.0)$ & $86(75.4)$ & $28(24.6)$ & \\
\hline Birthweight, kg & & & & 0.003 \\
\hline$<1$ & $17(7.6)$ & $8(47.0)$ & $9(53.0)$ & \\
\hline $1-1.5$ & $102(45.6)$ & $71(69.5)$ & $31(30.4)$ & \\
\hline$>1.5$ & $105(46.8)$ & $87(82.8)$ & $18(17.2)$ & \\
\hline Gender & & & & 0.103 \\
\hline Female & $136(60.7)$ & $106(78.0)$ & $30(22.0)$ & \\
\hline Male & $88(39.3)$ & $60(68.2)$ & $28(31.8)$ & \\
\hline Gestational age, weeks & & & & 0.063 \\
\hline$\leq 28$ & $22(9.80)$ & $15(68.2)$ & $7(31.8)$ & \\
\hline $29-30$ & $58(25.9)$ & $37(63.8)$ & $21(36.2)$ & \\
\hline $31-36$ & $137(61.2)$ & $107(78.1)$ & $30(21.9)$ & \\
\hline$\leq 37$ & $7(3.1)$ & $7(100)$ & 0 & \\
\hline Apgar score (5 min) & & & & 0.347 \\
\hline$<5$ & $17(7.6)$ & $15(88.2)$ & $2(11.8)$ & \\
\hline $5-7$ & $69(30.8)$ & $49(71.0)$ & $20(29.0)$ & \\
\hline$>7$ & $138(61.6)$ & $102(73.9)$ & $36(26.1)$ & \\
\hline Resuscitation & & & & 0.805 \\
\hline None & $159(70.9)$ & $118(74.2)$ & $41(27.8)$ & \\
\hline Stimulation & $30(13.4)$ & $21(70.0)$ & $9(30.0)$ & \\
\hline Bagging $/ \mathrm{O}_{2}$ & $35(15.7)$ & $27(77.0)$ & $8(23.0)$ & \\
\hline Antibiotics & & & & 0.743 \\
\hline None & $9(4.0)$ & $7(77.0)$ & $2(23.0)$ & \\
\hline 1st-line & $124(55.3)$ & $95(67.6)$ & $29(23.4)$ & \\
\hline 2nd-line & $66(29.4)$ & $47(71.2)$ & $19(28.8)$ & \\
\hline 3rd-line & $25(11.3)$ & $17(68.0)$ & $8(32.0)$ & \\
\hline Respiratory support & & & & 0.188 \\
\hline Not using & $76(34.0)$ & $62(81.6)$ & $14(18.4)$ & \\
\hline $\mathrm{NPO}_{2}$ only & $49(21.8)$ & $37(75.5)$ & $12(24.5)$ & \\
\hline CPAP & $64(28.5)$ & $42(65.6)$ & $22(34.4)$ & \\
\hline IPPV & $35(15.6)$ & $25(71.4)$ & $10(28.6)$ & \\
\hline Neonatal sepsis & & & & 0.231 \\
\hline No & $25(11.0)$ & $21(84.0)$ & $4(16.0)$ & \\
\hline Yes & $199(89.0)$ & $145(72.8)$ & $54(27.2)$ & \\
\hline Axillary temperature, ${ }^{\circ} \mathrm{C}$ & & & & 0.005 \\
\hline Normal (36.0 - 37.5) & $198(88.3)$ & $154(77.8)$ & $44(22.2)$ & \\
\hline Abnormal $(<36.0$ or $>37.5)$ & $26(11.7)$ & $12(46.0)$ & $14(54.0)$ & \\
\hline Blood glucose, mmol/L & & & & 0.035 \\
\hline Normal (2.6 - 7.5) & $180(80.3)$ & $141(78.3)$ & $39(21.7)$ & \\
\hline Abnormal $(<2.5$ or $>7.5)$ & $44(19.7)$ & $25(56.8)$ & $19(43.2)$ & \\
\hline
\end{tabular}

neonatal jaundice ( $10.6 \%$ v. $8.0 \%)$ and feeding problems ( $8.6 \%$ v. $7.0 \%)$ were equally common in both sites.

In the present study, babies were admitted to $\mathrm{KMC}$ once they were stable, at a median age of 9.5 days. The proportion of babies with a poor outcome was highest among those admitted after seven days of age although this was not statistically significant, which was possibly due to late admission to KMC associated with a more immature newborn or a complicated course in the nursery. In Zimbabwe, Kambarami et al ${ }^{[18]}$ also reported a higher rate of readmission to the nursery among older babies, especially those admitted after 14 days of age. Just over a third (38.4\%) of babies had a five-minute Apgar score below seven, which corresponds to less than a third (29.1\%) of babies requiring resuscitation. This finding had no impact on the outcome of babies admitted to KMC. Birthweight and gestational age were both associated with poor outcome. Although the poorer outcome seen in babies with a gestational age under 31 weeks was not significant, there was a significant association between both birthweight and weight on admission below $1500 \mathrm{~g}$ and a poor outcome. These findings are similar to those reported by Kambarami et al. ${ }^{[18]}$ in Zimbabwe. ${ }^{[18]}$ 


\begin{tabular}{|c|c|c|c|c|}
\hline Indicator & Total $(N=224), n(\%)$ & Discharged $(N=166), n(\%)$ & Poor outcome $(N=58), n(\%)$ & $p$-value \\
\hline Maternal age, years & & & & 0.645 \\
\hline$<19$ & $24(10.7)$ & $16(66.6)$ & $8(33.4)$ & \\
\hline $19-35$ & $186(83.0)$ & $139(74.7)$ & $47(25.3)$ & \\
\hline$>35$ & $14(6.30)$ & $11(78.6)$ & $3(21.5)$ & \\
\hline Parity & & & & 0.292 \\
\hline Primigravida & $82(36.6)$ & $56(68.3)$ & $26(31.7)$ & \\
\hline Multigravida & $139(62.0)$ & $108(77.7)$ & $31(22.3)$ & \\
\hline Grand multi & $3(1.40)$ & $2(66.6)$ & $1(33.4)$ & \\
\hline Antenatal visit & & & & 0.317 \\
\hline Booked & $194(86.6)$ & $146(75.2)$ & $48(24.8)$ & \\
\hline Unbooked & $30(13.4)$ & $20(66.6)$ & $10(33.4)$ & \\
\hline HIV status & & & & 0.017 \\
\hline Negative & $145(64.7)$ & $100(68.8)$ & $45(31.2)$ & \\
\hline Positive & $79(35.5)$ & $66(83.5)$ & $13(16.5)$ & \\
\hline Antenatal problem & & & & 0.137 \\
\hline None & $71(31.6)$ & $56(78.8)$ & $15(21.2)$ & \\
\hline Pregnancy-related & $148(66.0)$ & $108(73.0)$ & $40(27.0)$ & \\
\hline Chronic disease & $5(2.4)$ & $2(40.0)$ & $3(60.0)$ & \\
\hline
\end{tabular}

Table 3. Newborns' characteristics during admission to kangaroo mother care unit

\begin{tabular}{|c|c|c|c|c|}
\hline Indicator & Total $(N=224), n(\%)$ & Discharge $(N=166), n(\%)$ & Poor outcome $(N=58), n(\%)$ & $p$-value \\
\hline Age on admission, days & & & & 0.423 \\
\hline $1-7$ & $101(45.0)$ & $79(78.2)$ & $22(21.8)$ & \\
\hline $8-14$ & $76(34.0)$ & $53(69.7)$ & $23(30.3)$ & \\
\hline$\geq 15$ & $47(21.0)$ & $34(72.3)$ & $13(27.7)$ & \\
\hline Weight on admission, $\mathrm{kg}$ & & & & 0.000 \\
\hline $1-1.25$ & $20(9.0)$ & $11(55.0)$ & $9(45.0)$ & \\
\hline$>1.25-1.5$ & $62(27.6)$ & $38(61.3)$ & $24(38.7)$ & \\
\hline$>1.5-1.57$ & $87(38.8)$ & $66(75.8)$ & $21(24.2)$ & \\
\hline$>1.57$ & $55(24.6)$ & $51(92.7)$ & $4(7.30)$ & \\
\hline Problem on admission & & & & 0.206 \\
\hline Weight gain & $7(3.2)$ & $6(85.7)$ & $1(14.3)$ & \\
\hline Prematurity-related & $96(42.8)$ & $76(79.0)$ & $20(21.0)$ & \\
\hline Sepsis & $121(54.0)$ & $84(69.4)$ & $37(30.6)$ & \\
\hline Axillary temperature, ${ }^{\circ} \mathrm{C}$ & & & & 0.008 \\
\hline Normal (36.0 - 37.5) & $217(96.8)$ & $164(75.6)$ & $53(24.4)$ & \\
\hline Abnormal $(<36.0->37.5)$ & $7(3.20)$ & $2(28.6)$ & $5(71.4)$ & \\
\hline Blood glucose, $\mathrm{mmol} / \mathrm{L}$ & & & & 0.006 \\
\hline Normal $(2.6-7.5)$ & $213(95.0)$ & $163(76.5)$ & $50(23.5)$ & \\
\hline Abnormal $(<2.6$ or $>7.5)$ & $11(5.0)$ & $3(27.3)$ & $8(72.7)$ & \\
\hline New problem in KMC & & & & 0.000 \\
\hline Weight gain & $186(83.0)$ & $149(80.2)$ & $37(19.9)$ & \\
\hline Food intolerance & $17(7.6)$ & $8(47.0)$ & $9(53.0)$ & \\
\hline Anaemia & $17(7.6)$ & $7(41.2)$ & $10(58.8)$ & \\
\hline Other & $4(1.8)$ & $2(50.0)$ & $2(50.0)$ & \\
\hline
\end{tabular}

Most babies received antibiotics (96\%) and some form of respiratory support (66\%) prior to admission to KMC but this was not associated with a poorer outcome. A clinical diagnosis of sepsis was more common in the nursery than the KMC unit ( $89 \%$ v. $54 \%$ ) but this had no association with the outcome in the KMC unit. An abnormal temperature was more common in the nursery than in the KMC unit (11.7\% v. 3.2\%) although in both sites this was associated with a statistically significant increase in poor outcome from KMC.
A similar finding occurred with abnormal blood glucose levels, which were more common in the nursery but statistically significant in both the nursery and KMC unit. Both Cattaneo et al. ${ }^{[19]}$ and Rahman et al. ${ }^{[20]}$ reported a higher incidence of abnormal temperature in the nursery than the KMC unit, although neither reported on the association of this observation with the outcome of newborns.

One in $10(10.7 \%)$ babies admitted to KMC had a teenage mother, which is a lower proportion than the $17.6 \%$ of teenage mothers in the 
Table 4. Reasons for poor outcomes

\begin{tabular}{llll}
\hline Reason & $\begin{array}{l}\text { Total } \\
(N=58), \\
n(\%)\end{array}$ & $\begin{array}{l}\text { Readmission } \\
(N=51), n(\%)\end{array}$ & $\begin{array}{l}\text { Death } \\
(N=7),\end{array}$ \\
\hline Maternal cause & $1(1.7)$ & $1(2.0)$ & 0 \\
$\begin{array}{l}\text { Feed intolerance } \\
\text { Aspiration }\end{array}$ & $5(8.6)$ & $5(9.8)$ & 0 \\
pneumonia & $4(6.9)$ & $1(2.0)$ & $3(42.8)$ \\
Neonatal sepsis & $32(55.2)$ & $31(60.6)$ & $1(14.3)$ \\
Neonatal jaundice & $6(10.3)$ & $6(11.8)$ & 0 \\
Anaemia & $6(10.3)$ & $6(11.8)$ & 0 \\
Apnoea only & $2(3.5)$ & 0 & $2(28.6)$ \\
Other & $2(3.5)$ & $1(2.0)$ & $1(14.3)$ \\
*For readmission/death. & & &
\end{tabular}

general newborn population in KZN reported in the District Health Barometer. ${ }^{[9]}$ This difference is possibly because babies of teenage mothers have a higher mortality rate ${ }^{[10]}$ and did not survive long enough to be admitted to KMC, as $75 \%$ of newborn deaths occur within the first week of life and 55\% of babies in this study were admitted to KMC after seven days of age. ${ }^{[3]}$

The higher proportion of newborns admitted to KMC with poor outcomes whose mothers were primigravid, grand multiparous or unbooked is similar to Onyema et al'. ${ }^{[11]}$ experience in Nigeria. Newborns who were HIV exposed had a better outcome, which is contrary to other reports from KZN where Naidoo et al. ${ }^{[12]}$ found a worse outcome with HIV exposure; and Luthuli et al.$^{[13]}$ reported no difference in outcome in very-low birthweight babies (VLBW) in association with maternal HIV status. This finding may relate to sample selection as only babies who survived to be admitted to KMC were included in this study. It is possible that a higher proportion of HIV-unexposed babies were admitted to KMC as the HIV-exposed babies possibly did worse in the nursery and did not survive to be transferred to KMC.

Two-thirds (66\%) of babies admitted to KMC had mothers with pregnancy-related complications, and a quarter (24\%) had mothers with chronic medical conditions during their antenatal period. Pregnancy-related problems included prolonged rupture of membranes, oligohydramnios, antepartum haemorrhage, gestational diabetes or pregnancy-induced hypertension. Khashu et al. ${ }^{[14]}$ identified the same risk factors while Shapiro-Mendoza et al. ${ }^{[15]}$ identified maternal medical conditions as the greatest risk factor for newborn morbidity, especially intrauterine growth retardation.

Half (51\%) of the mothers had a caesarean section, which is higher than the provincial average for KZN of $30.7 \%^{[16]}$ but similar to the 42.4\% reported from the Lower Umfolozi War Memorial District Hospital. ${ }^{[17]}$ However, the mode of delivery did not influence the newborn outcome. Although there was a predominance of female babies and they had a slightly lower proportion with a poor outcome, this factor was not statistically significant.

The present study highlights the importance of admission weight, abnormal observations during admission to KMC, and the emergence of new problems as markers of a poor newborn outcome.

\section{Conclusion}

Three-quarters of babies admitted to KMC had an uneventful course and were discharged home, while $26 \%$ of babies experienced poor outcomes, with seven dying in the KMC units and 51 requiring readmission to the nursery. Poor outcomes were associated with a birthweight or weight on admission to KMC below $1500 \mathrm{~g}$ and with an abnormal temperature or blood glucose level during admission to the nursery or KMC unit.

Admission criteria may need to be reviewed and responses to abnormal observations strengthened in line with individual KMC unit resources.

Declaration. The manuscript was submitted in partial fulfilment of the requirements for the MMed degree of the University of KwaZulu-Natal.

Acknowledgements. We thank the staff and patients in the KMC units at both RKKH and MGMH hospitals.

Author contributions. WB was responsible for conceptualisation of the research, data collection and analysis and preparation of the manuscript; NK was co-supervisor and responsible for conceptualisation of the research and review of the draft manuscript; NHM was the principal supervisor and contributed to the conceptualisation of the research, data analysis and preparation of the manuscript.

Funding. None.

Conflicts of interest. None.

1. Bamford LJ, McKerrow NH, Barron P, Aung Y. Child mortality in South Africa: Fewer deaths, but better data are needed. S Afr Med J 2018;108(3):25-32. https://doi.org/10.7196/SAMJ.2017.v108i3b.12779

2. National Department of Health. National perinatal morbidity and mortality committee (NaPeMMCo) annual report 2018. Pretoria: NDoH, 2018.

3. United Nations Inter-agency Group for Child Mortality Estimation. Levels \& Trends in Child Mortality: Report 2019. Estimates developed by the United Nations Inter-agency Group for Child Mortality Estimation. New York: United Nations Children's Fund; 2019.

4. Howson CP, Kinney MV, McDougall L, Lawn JE. Born too soon: Preterm birth matters. Reproductive Health 2013;10(Suppl 1):S1. http://www. reproductive-health-journal.com/content/10/S1/S1

5. Charpak N, Ruiz-Pelaez JG, De Calume Figueroa Z. Current knowledge of kangaroo mother intervention. Curr Opin Pediatr 1996;8:108-112. https:// doi.org/10.1097/00008480-199604000-00004

6. Cattaneo A, Davanzo R, Uxa F, Tamburlini G. Recommendations for the implementation of kangaroo mother care for low birth weight infants. International network on kangaroo mother care. Acta Paediatr 1998;87(4):440-445. https://doi.org/10.1111/j.1651-2227.1998.tb01475.x

7. Nyqvist KH, Anderson GC, Bergman N, et al. Towards universal kangaroo mother care: Recommendations and report from the first European conference and seventh international workshop on kangaroo mother care. Acta Paediatrica 2010;99(6):820-826. https://doi.org/10.1111/j.1651 2227.2010.01787.x

8. Rodriguez AN, Nel M, Dippenaar H, Prinsloo EA. Good short-term outcome of kangaroo mother care in low birth weight infants in a rural South African hospital. S Afr Family Practice 2007;49(5):15-15c. https://www.ajol.info/ index.php/safp/article/view/13363/64214

9. Massyn N, Pillay Y, Padarath A. District Health Barometer. Health Systems 2017/2018. Durban: Health System Trust; 2019.

10. Neal S, Channon AA, Chintsanya J. The impact of young maternal age at birth on neonatal mortality: Evidence from 45 low and middle income countries. PLoS ONE 2018;13(5):e0195731. https://doi.org/10.1371/journal. pone. 0195731

11. Onyema MC, Obi VO, Nwafor JI, et al. Profiling the socio-demographic characteristics and outcome of preterm delivery in Alex Ekwueme Federal University Teaching Hospital Abakaliki. Open J Obstetr Gynecol 2019;9(8):1168. https://doi.org/10.4236/ojog.2019.98113

12. Naidoo M, Sartorius B, Tshimanga-Tshikala G. Maternal HIV infection and preterm delivery outcomes at an urban district hospital in KwaZulu-Natal 2011. Southern Afr J Infect Dis 2016;31(1):25-28. https://doi.org/10.1080/2 3120053.2016 .1118838

13. Luthuli NP, McKerrow NH. Short-term outcomes of infants with an extremely low birth weight in a resource-limited neonatal intensive care unit, Grey's Hospital, KwaZulu-Natal. S Afr J Child Health 2019;13(3):120-124. https:// doi.org/10.7196/SAJCH.2019.v13i3.1575

14. Khashu M, Narayanan M, Bhargava S, Osiovich H. Perinatal outcomes associated with preterm birth at 33 to 36 weeks' gestation: A populationbased cohort study. Pediatr 2009;123(1):109-113. https://doi.org/10.1542/ peds.2007-3743

15. Shapiro-Mendoza CK, Tomashek KM, Kotelchuck M, et al. Effect of latepreterm birth and maternal medical conditions on newborn morbidity risk. Pediatr 2008;121(2):e223-e232. https://doi.org/10.1542/peds.2006-3629

16. Massyn N, Padarath A, English R, editors. Maternal, Child and Women's Health Barometer 2016. Durban: Health Systems Trust; 2018. 
17. Makhanya V, Govender L, Moodley J. Utility of the Robson Ten Group Classification System to determine appropriateness of caesarean section at a rural regional hospital in KwaZulu-Natal, South Africa. S Afr Med J 2015;105(4):292-295. https://doi.org/10.7196/SAMJ.9405

18. Kambarami RA, Chidede O, Kowo DT. Kangaroo care for well low birth weight infants at Harare Central Hospital Maternity Unit--Zimbabwe. Central Afr J Med 1999;45(3):56-59. https://doi.org/10.4314/cajm.v45i3.8454

19. Cattaneo A, Davanzo R, Worku B, et al. Kangaroo mother care for low birthweight infants: A randomised controlled trial in different settings. Acta Paediatr 1998;87(9):976-985. https://doi.org/10.1111/j.1651-2227.1998.tb01769.x
20. Rahman M, Chowdhury MA, Hoque M, Jahan N, Shaha LC. Kangaroo mother care for low birth weight babies: A randomised controlled trial in a tertiary care hospital of Bangladesh. J Pediatr Neonat Care 2017;7(2):00285.

21. Blencowe H, Molyneux EM. Setting up kangaroo mother care at Queen Elizabeth Central Hospital, Blantyre-a practical approach. Malawi Med 2005; 17(2):39-42.

Accepted 2 October 2020 